\title{
Sur la géométrie différentielle des surfaces reglées.
}

\author{
Othon Pylarinos (à Athèneg, Grèce) (*)
}

Resumé. - Est donné dans l'introduction.

La courbure normale et la torsion géodésique de la ligne de striction d'une surface reglée gauche de l'espace euclidien à trois dimensions sont des invariants métriques de la surface. Par ailleurs ces deux grandeurs sont des fonctions de la variable aux valeurs de laquelle correspondent les points de la ligne de striction; aussi sont-elles liées en général par une relation et les surfaces reglées gauches sur lesquelles ces grandeurs sont liées par des relations de la mème forme ont des propriétés communes dont la recherche peut conduire à des resultats concernant la géométrie différentielle métrique des surfaces reglées, qui ne sont pas dépourvus d'intérêt.

Dans le présent article, consacré à la recherche indiquée, j'établis certains théorèmes concernant les surfaces reglées gauches, sur chacune desquelles la courbure normale et la torsion géodésique de sa ligne de striction vérifient une relation algébrique du premier ou du second degré à coefficients constants. En outre, après avoir démontré que, lorsque la courbure et la torsion géodésiques d'une trajectoire orthogonale des génératrices d' une surface reglée gauche vérifient une relation algébrique à coefficients constants, la courbure et la torsion géodésiques de toute autre trajectoire orthogonale des génératrices de la surface sont nécessairement liées par une relation algébrique à coefficients constants, je parviens à l'aide des théorèmes établis, à la détermination de certaines classes de surfaces reglées, sur lesquelles la courbure et la torsion géodésiques de chaque trajectoire orthogonale de leurs génératrices vérifient une relation algébrique du second degré au plus à coefficients qui sont constants sur elle, mais qui varient en général de l'une à l'autre de ces courbes. Je montre ainsi que la courbure et la torsion géodésiques de chaque trajectoire orthogonale des génératrices de la surface engendrée par les normales principales d'une courbe de Bertrand ou d'une courbe de Mannheim ou d'une hélice cylindrique, ainsi que d'une surface

(*) Entrata in Redazione il 14 giugno 1970. 
gauche à paramètre de distribution constant ou de la surface engendrée par les binormales d'une courbe gauche vérifient une relation algébrique $d u$ second degré au plus à coefficients constants sur elle; ces coefficients, si l'on excepte le cas des surfaces à paramètre de distribntion constant, varient de l'une à l'autre de ces courbes.

I.

1. Considérons sur une surface reglée gauche réelle ds l'espace euclidien à trois dimensions une portion $R$ dont le paramètre de distribution est $\neq 0$ sur toutes les génératrices.

Soit

$$
\vec{\rho}=\bar{\rho}(u)
$$

l'équation vectorielle par rapport au système de coordonnées choisi dans l'espace de la ligne de striction $C$ de $R$, lieu des points centraux des génératrices de cette surface, le paramètre $u$ étant l'arc de $O$.

Si $\bar{e}(u), \bar{n}(u), \bar{z}(u)=\bar{e} \wedge \bar{n}$ sont les vecteurs unitaires qui déterminent les sens positifs sur la génératrice $e$ de $R$ issue du point courant $K(u)$ de $C$, de la normale centrale $n$ de $R$ en $K$ et de la tangente centrale $z$ de $R$ en ce même point, e.à.d. de la tangente à $R$ en $K$ perpendiculaire à la génératrice $e$, on a - comme on sait $(4$, p. 145) - pour les dérivées des $\bar{e}, \bar{n}, \bar{z}$ par rapport à la variable $u$ les formules

$$
\dot{\bar{e}}=x \bar{n}, \quad \dot{\bar{n}}=-x \bar{e}+\sigma \bar{z}, \quad \dot{\bar{z}}=-\sigma \bar{n}\left({ }^{1}\right),
$$

où

$$
x=\varepsilon|\dot{\bar{e}}|, \quad \sigma=\frac{(\bar{e} \wedge \dot{\bar{e}} \mid \times \dot{\bar{e}}}{|\dot{\bar{e}}|^{2}}
$$

et $\varepsilon=+1$ ou -1 suivant que le produit mixte $\dot{\vec{\rho}} \wedge \bar{e}) \times \dot{\bar{e}}$, qui, d'après l'hypothèse faite pour le paramètre de distribution de $R$, est $\neq 0$ pour toutes les valeurs de $u$ correspondant aux points de $C$, est $>$ ou $<0$.

Les droites $e, n, z$ issues du point $K(u)$ de la courbe $O$, sur les directions desquelles les sens positifs se déterminent par les vecteurs unitaires $\bar{e}, \bar{n}, \bar{z}$

${ }^{(1)}$ Les points désignent les dérivées par rapport à la variable $u$. On suppose que les operations de dérivation qui seront faites, dans ce qui suit, sont légitimes dans les intervalles oonsiderés. 
respectivement, sont les axes d' un trièdre trirectangle orienté $[K ; \bar{e}, \bar{n}, \bar{z}]$; ce trièdre est appelé, dans ce qui suit, trièdre central associé à la surface.

Par ailleurs la dérivée $\dot{\bar{\rho}}$ au point $K(u)$ de la courbe $C$ est un vecteur unitaire, puisque $u$ est l'arc de $C$. Ce vecteur, étant parallèle à la tangente à $C$ en $K$, est parallèle au plan tangent $[K ; \bar{e}, \bar{z}]$ à $R$ en ce point. On peut donc le mettre sous la forme

$$
\dot{\bar{\rho}} \equiv \bar{t}=\bar{e} \cos \varphi+\bar{z} \sin \varphi,
$$

où $\varphi$ est l'angle orienté $(\bar{\varepsilon}, \bar{l})$ sur le plan $[K ; \bar{e}, \bar{z}]$.

Les trois grandeurs $\chi, \sigma, \varphi$ sont des fonctions de l'arc $u$ de la ligne de striction $C$ de $R$ définies, grâce à l'hypothèse faite pour le paramètre de distribution de $R$, dans l'intervalle de valeurs de $u$ correspondant aux points de la courbe $C$ ou - ce qui revient au même-aux géneratrices de $R$. Ces trois fonctions $x(u), \sigma(u), \varphi(u)$, dont les valeurs sur chaque génératrice de $R$ sont appelées par E. KRUPPA (3, p. 63), courbure, torsion et striction de la surface sur cette génératrice sont avec l'arc $u$ de $C$ des invariants métriques de $R$ et ce sont précisément les invariants métriques de la surface, qui ont été choisis comme fondamentaux dans la géométrie différentielle métrique des surfaces reglées fondée par G. Sannia [5] et E. Kruppa [4].

Deux autres invariants métriques de la surface sont la courbure normale $x_{n}$ et la torsion géodésique $\sigma_{g}$ de sa ligne de striction. Ces denx grandeurs sont des fonctions des trois invariants fondamentaux de la surface.

En effet, d'après des formules connues $(3$, p. 73$)$, on a

$$
x_{n}=x \cos \varphi-\sigma \sin \varphi, \quad \sigma_{g}=x \sin \varphi+\sigma \cos \varphi .
$$

RemarQue. - La surface $R$ coupe sous un angle droit - comme on le voit aussitôt - la surface $R_{n}$ engendrée par les normales centrales de $R$ tont le long de sa ligne de striction $C$ qui évidemment est une trajectoire orthogonale des génératrices de $R_{n}$.

Il en résulte que la courbure normale $x_{n}$ et la torsion géodésique $\sigma_{g} d \theta$ la courbe $C$ considérée comme une courbe tracée sur la surface $R$, sont en chaque point de $C$ la premiére égale en valeur absolue à la courbure géodé. sique $x_{g}^{\prime}$ et la seconde égale à la torsion géodésique $\sigma_{g}^{\prime}$ de $C$ considérée comme une courbe tracée sur la surface $R_{n}$.

On aura donc en chaque point de $C$

$$
x_{g}^{\prime}=\varepsilon^{\prime} x_{n}, \quad \sigma_{g}^{\prime}=\sigma_{g},
$$

où $\varepsilon^{\prime}=+1$ ou -1 et on pent choisir le sens positif sur la direction de la normale à $R_{n}$ de manière que l'on ait 


$$
x_{g}^{\prime}=x_{n}, \quad \sigma_{g}^{\prime}=\sigma_{g} .
$$

2. L'équation (1.1) de la courbe $C$, gràce à (1.4), peut s'écrire

$$
\bar{p}=\int(\bar{e} \cos \varphi+\bar{z} \sin \varphi) d u
$$

et l'équation (vectorielle) de la surface $R$, si l'on choisit sur elle sa ligne de striction $C$ comme courbe directrice, peut se mettre sous la forme

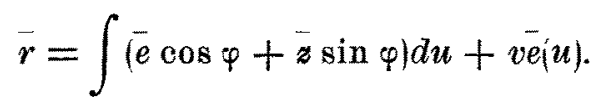

Cela posé, soit $R^{\prime}$ une surface reglée gauche coupant $R$ sous un angle droit tout le long d'une trajectoire orthogdnale $C^{\prime}$ des génératrices de $R$.

$\mathrm{Si}$

$$
\bar{\rho}^{\prime}=\bar{\rho}^{\prime}(u)=\bar{\rho}(u)+v^{\prime}(u) \bar{e}(u)
$$

est l'équation de la courbe $C^{\prime}$, la normale à $R$ au point courant $K^{\prime}(u)$ de cette courbe est parallèle au vecteur

$$
\bar{n}^{\prime}(u)=\frac{\dot{\bar{\rho}}}{|\dot{\bar{\rho}}|} \wedge \bar{e}=\bar{t}^{\prime} \wedge \bar{e}
$$

qui est un vecteur unitaire, puisque les vecteurs unitaires $\frac{\dot{\overline{\rho^{\prime}}}}{\dot{\dot{\rho^{\prime}}} \mid}=\overline{t^{\prime}}, \bar{e}$, d'après
$l^{\prime}$ hypothèse faite pour la courbe $C^{\prime}$, sont orthogonaux.

La génératrice $e^{\prime}$ de la surface $R^{\prime}$ issue du point $K^{\prime}(u)$ de $O^{\prime}$ est située sur le plan $\left[K^{\prime} ; \overline{t^{\prime}}, \overline{n^{\prime}}\right]$ perpendiculaire en $K^{\prime}$ à la génératrice de $R$, issne de ce point et, par conséquent, elle est nécessairement parallèle à un vecteur unitaire de la forme

$$
\overline{e^{\prime}}=\overline{t^{\prime}} \cos \theta+\overline{n^{\prime}} \sin \theta
$$

Pour que la courbe $C^{\prime}$ soit en plus la ligne de striction de la surface $R^{\prime}$, il faut et il suffit, comme on sait, que l'on ait en chaque point de $C^{\prime}$

$$
\overline{t^{\prime}} \times \dot{e^{\prime}}=0 \text {. }
$$

Mais de (2.4) on a

$$
\dot{\overline{e^{\prime}}}=\dot{\overline{t^{\prime}}} \cos \theta+\dot{\bar{n}^{\prime}} \sin \theta+\dot{\theta}\left(-\overline{t^{\prime}} \sin \theta+\bar{n}^{\prime} \cos \theta\right)
$$


et la condition (2.5), si l'on tient compte du fait que l'on a $\overline{t^{\prime 2}} \equiv 1, \overline{t^{\prime}} \times \dot{\overline{t^{\prime}}} \equiv 0$ et, gràce aux hypothèses faites, $\bar{t}^{\prime} \times \bar{n}^{\prime}=0$, sin $\theta \neq 0$, devient

$$
\dot{\theta}-\overline{t^{\prime}} \times \dot{\bar{n}}^{\prime}=0 \text {. }
$$

Le vectenr $\overline{e^{\prime}}(u)(2.4)$ pour chaque fonction $\theta(u)$ satisfaisant à l'équation différentielle (2.6) détermine $\infty^{1}$ droites, issues des points de la courbe $C^{\prime}$, qui engendrent une surface reglée gauche $R^{\prime}$ coupant $R$ le long de $C^{\prime}$ sous un angle droit et admettant cette courbe comme ligne de striction.

Mais la courbe $C^{\prime}$ est une trajectorie orthogonale des génératrices de $R$ choisie au hasard. Par ailleurs les considérations précédentes n'exigent pas que la surface reglêe $R$ soit ganche.

On peut donc énoncer le

Théoǹme I. - Chaque trajectoire orthogonale des génératrice d' une surface reglée est la ligne de striction de $\infty^{1}$ surfaces reglées gauches qui admettent les génératrices de la surface comme normales centrales.

Par ailleurs l'équation vectorielle d' nne surface reglée gauche $R_{1}$ repré. sentée sur la surface gauche $R$ définie par l'équation (2.2) de manière que les points homologues des denx surfaces admettent les mêmes coordonnées curvilignes $u$, $v$, est nécessairemenf - comme on sait $(5 ;$ p. 45) - de la forme

$$
\bar{r}_{1}=\int(\dot{e} \cos \varphi+\bar{z} \sin \varphi) d u+\overline{\beta n}+v_{1}(v)(\bar{e} \cos \omega+\bar{z} \sin \omega)
$$

où

$$
\beta=\text { Cte }, \quad \omega=\operatorname{Cte}(-\pi<\omega \leq \pi),
$$

la fonction $v_{1}(v)$ étant arbitraire, lorsque, dans cette représentation, les génératrices des deux surfaces ainsi que leurs lignes de striction se correspondent, les deux surfaces ayant en outre des normales coïncidantes en chaque couple de points homologues de leurs lignes de strietion.

L'équation (2.7), pour chaque système de valeurs de paramètres $\beta$, $\omega$ qui $y$ figurent, détermine une surface reglée ganche. Cette surface, lorsque $\beta$ est $\neq 0$, si $\omega=0$ ou $\pi$, ne coincide pas avec la surface $R$ et les denx surfaces, étant représantées l' une sur l'autre de la manière indiquée, admettent des normales centrales coïncidantes aux points homologues de leurs lignes de striction.

Il en rósulte, eu égard au fait que $R$ est une surface reglée gauche choisie au hasard, $q u$ 'à chaque surface reglée gauche on peut faire associer $\infty^{2}$ surfaces reglées gauches dont les normales centrales coüncident aveo les normales centrales de cette surface. 
$L^{\prime}$ ensemble de surfaces représentées sur une surface reglée gauche de la manière indiquée est appelé, dans ce qui suit, ensemble $(N)$ associé $\grave{\alpha}$ celte surface.

L' ensemble $(N)$ associé à la surface considérée $R$ est défini par l'équation (2.7). Les surfaces de cet ensemble, la surface $R y$ comprise, correspondent aux systèmes de valeurs des paramètres $\beta$, w qui figurent dans cette équation, appartenant respectivement à l'intervalle ouvert $(-\infty,+\infty)$ et à l'intervalle supérieurement fermé $(-\pi,+\pi)$.

3. La génératrice d'une surface $R_{1}$ de l'ensemble $(N)$ associé à la surface $R$, homologue de la génératrice de $R$ issue du point courant $K(u)$ de sa ligne de striction $C$, est nécessairement parallèle an plan tangent à $R$ en $K$ et, d'après (2.8), elle est invariablement liée avec le trièdre central associè à $R$.

Cela étant, on peut choisir le sens positif sur la direction de cette droite de manière que les sens positifs sur la direction commune des normales coüncidantes des surfaces $R, R_{1}$ en chaque couple de points homologues de leurs lignes de striction coïncident.

On aura ainsi, en désignant par $\bar{e}_{1}, \bar{n}_{1}, \bar{z}_{1}$ les vecteurs unitaires qui déterminent les sens positifs sur les directions de axes du trièdre central associé $\dot{a} R_{1}$ au point $K_{1}$ de sa ligne des striction $C_{1}$ homologue du point courant $K(u)$ de la ligne de striction $C$ de $R$, les relations:

$$
\vec{e}_{1}=\bar{e} \cos \omega+\bar{z} \sin \omega, \quad \bar{n}_{1}=\bar{n}, \quad \bar{z}_{1}=\bar{e}_{1} \wedge \bar{n}_{1}=-\bar{e} \sin \omega+\bar{z} \cos \omega .
$$

Par ailleurs la ligne de striction $C_{1}$ de $R_{1}$ est définie, d'après (2.7), par l'équation

$$
\bar{\rho}_{1}=\bar{\rho}_{1}(u)=\int(\bar{e} \cos \varphi+\bar{z} \sin \varphi) d u+\beta \bar{n}(u),
$$

où, d'après $(2.8), \beta$ est une constante.

En différentiant (3.2) par rapport à $u$ et en faisant usage des (1.2), il vient

$$
\frac{d \overline{\rho_{1}}}{d u}=\bar{e}(\cos \varphi-\beta x)+\bar{z}(\sin \varphi+\beta \sigma) \text {. }
$$

On aura done, en désignant par $d u_{1}$ l'arc élémentaire de la courbe $C_{1}$ en son point $K_{1}$ homologue du point courant $K(u)$ de la courbe $C$,

$$
\left(\frac{d u_{1}}{d u}\right)^{2}=\left(\frac{d \bar{\rho}_{1}}{d u}\right)^{2}=(\cos \varphi-\beta x)^{2}+(\sin \varphi+\beta \sigma)^{2}
$$


Cette relation, à l'aide des (1.5), affecte la forme

$$
\left(\frac{d u_{1}}{d u}\right)^{2}=1-2 \beta x_{n}+\beta^{2}\left(x_{n}^{2}+\sigma_{g}^{2}\right)=\frac{1}{\lambda^{2}}
$$

ce qui montre que le rapport des ares élémentaires $d u_{1}$, $d u$ des courbes $C_{1}$, $C$ en leurs points homologues $K_{1}, K$ est une fonction algébrique de la courbure normale $x_{n}$ et de la torsion géodésique $\sigma_{g}$ de la courbe $O$, le coefficient $\beta$, qui $y$ figure, étant une costante égale en valeur absolue à la distance constante, d'après (2.8), des points homologues des deux courbes.

De même en différentiant la première et la troisième relation (3.1) par rapport à $i \xi$ et en faisant usage des (1.2), il vient

$$
\dot{\bar{e}}_{1}=(x \cos \omega-\sigma \sin \omega) \bar{n}, \quad \dot{\bar{z}}_{1}=-(x \sin +\sigma \cos \omega) \bar{n} .
$$

Si l'on désigne maintenant par $\chi_{1}, \sigma_{1}, \varphi_{1}$ la courbure, la torsion et la striction de la surface $R_{1}$ sur sa génératrice homologue de la génératrice courante de $R$ et que l'on tienne compte que, d'après des formules connues $(4$, p. 145), on a

$$
\frac{d \bar{e}_{1}}{d u_{1}}=x_{1} \bar{n}_{1}, \quad \frac{d \bar{z}_{1}}{d u_{1}}=-\sigma_{1} \bar{n}_{1}
$$

des ces formules, à l'aide des $(3.4)$ et $(3.5$, on parvient aux relations

$$
\begin{aligned}
& \frac{d \bar{e}_{1}}{d u_{1}}=x_{1} \bar{n}_{1}=(x \cos \omega-\sigma \sin \omega) \frac{d u}{d u_{1}} \bar{n}=\lambda(x \cos \omega-\sigma \sin \omega) \bar{n} \\
& \frac{d \bar{z}_{1}}{d u_{1}}=-\sigma_{1} \bar{n}_{1}=-(x \sin \omega+\sigma \cos \omega) \frac{d u}{d u_{1}} \bar{n}=-\lambda(x \sin \omega+\sigma \cos \omega) \bar{n}
\end{aligned}
$$

Mais, d'après la seconde relation $(3.1)$, on a $\bar{n}=\bar{n}_{1}$; on aura done

$$
x_{1}=\lambda(x \cos \omega-\sigma \sin \omega), \quad \sigma_{1}=\lambda(x \sin \omega+\sigma \cos \omega) .
$$

En outre on a

$$
\begin{aligned}
& \cos \varphi_{1}=\frac{d \bar{\rho}_{1}}{d u_{1}} \times \bar{e}_{1}=\frac{d u}{d u_{1}} \frac{d \bar{\rho}_{1}}{d u} \times{\overrightarrow{e_{1}}}_{1}=\lambda \frac{d \bar{\rho}_{1}}{d u} \times{\overline{e_{1}}}_{1} \\
& \sin \varphi_{1}=\frac{d \bar{\rho}_{1}}{d u_{1}} \times \bar{z}_{1}=\frac{d u}{d u_{1}} \frac{d \bar{\rho}_{1}}{d u} \times \bar{z}_{1}=\lambda \frac{d \bar{\rho}_{1}}{d u} \times \bar{z}_{1}
\end{aligned}
$$


puisque la tangente à la courbe $C_{1}$ en son point $K_{1}$ est évidemment située sur le plan tangent $\left[K_{1} ; \bar{e}_{1}, \bar{z}_{1}\right]$ à $R_{1}$ en ce même point.

Ces relations, si l'on $y$ remplace $\bar{e}_{1}, \bar{z}_{1}, \frac{d \bar{\rho}_{1}}{d u}$ par leurs valeurs $(3.1)$ et (3.3), prennent la forme

$$
\left\{\begin{array}{l}
\cos \varphi_{1}=\lambda(\cos \omega(\cos \varphi-\beta x)+\sin \omega(\sin \varphi+\beta \sigma)\} \\
\sin \varphi_{1}=\lambda\{-\sin \omega(\cos \varphi-\beta x)+\cos \omega(\sin \varphi+\beta \sigma)\} .
\end{array}\right.
$$

Enfin, si l'on tient compte du fait que la courbure normale $x_{1 n}$ et la torsion géodésique $\sigma_{1 g}$ de la ligne de striction $C_{1}$ de $R_{1}$ sont liées, d'après des formules connues $\left(3\right.$, p. 73 ), avec les invariants fondamentaux $x_{1}, \sigma_{1}, \varphi_{1}$ de la surface par les relations

$$
x_{1 n}=x_{1} \cos \varphi_{1}-\sigma_{1} \sin \varphi_{1}, \quad \sigma_{1 g}=x_{1} \sin \varphi_{1}+\sigma_{1} \cos \varphi_{1},
$$

on parvient en $y$ remplaçant $\chi_{1}, \sigma_{1}$ et $\cos \varphi_{1}, \sin \varphi_{1}$ par leurs valeurs (3.6) et (3.7) et en faisant usage des (1.5), aux relations

$$
\chi_{1 n}=\lambda^{2}\left\{x_{n}-\beta\left(\chi_{n}^{2}+\sigma_{g}^{2}\right)\right\}, \quad \sigma_{1 g}=\lambda^{2} \sigma_{g}
$$

et finalement, grâce à (3.4), aux formules

$$
x_{1 n}=\frac{x_{n}-\beta\left(x_{n}^{2}+\sigma_{g}^{2}\right)}{1-2 \beta x_{n}+\beta^{2}\left(x_{n}^{2}+\sigma_{g}^{2}\right)}, \quad \sigma_{1 g}=\frac{\sigma_{g}}{1-2 \beta x_{n}+\beta^{2}\left(x_{n}^{2}+\sigma_{g}^{2}\right)} .
$$

Mais, d'après la remarque finale du paragraphe 1 , la courbure normale et la torsion géodésique de la ligne de striction de la surface $R$, ainsi que de toute autre surface $R_{1}$ de l'ensemble $(N)$ associé à $R$, sont liées par les relations (1.6) avee la courbure et la torsion géodésiques de cotte courbe considérée comme une courbe tracée sur la surface $R_{n}$ engendrée par les normales centrales de $R$.

Cela étant, des deux formules (3.8), en désignant par $x_{g}^{\prime}, x_{I g}^{\prime} ; \sigma_{g}^{\prime}$, $\sigma_{1 g}^{\prime}$ les courbures et les torsions géodésiques des lignes de striction $C, C_{1}$ des surfaces $R, R_{1}$, considérées comme des courbes tracées sur la surface $R_{n}$, on obtient les relations

$$
x_{1 g}^{\prime}=\frac{x_{g}^{\prime}-\beta\left(x_{g}^{\prime 2}+\sigma_{g}^{\prime 2}\right)}{1-2 \beta x_{g}^{\prime}+\beta^{2}\left(x_{g}^{\prime 2}+\sigma_{g}^{\prime 2}\right)}, \quad \sigma_{1 g}^{\prime}=\frac{\sigma_{g}^{\prime}}{1-2 \beta x_{g}^{\prime}+\beta^{2}\left(x_{g}^{\prime 2}+\sigma_{g}^{\prime 2}\right)},
$$

le coefficient $\beta$ qui $y$ figure étant égal en valeur absolue à la distance constante, d'après (2.8), des deux points des courbes $C, C_{1}$ situés sur la même génératrice de la surface $R$. 
La ligne de striction $C_{1}$ de la surface $R_{1}$ est évidemment une trajectoire orthogonale des génératrices de la surface $R_{n}$ choisie au hasard et les consi. dérations précédentes concernant la courbure et la torsion géodesiques de cette courbe tracée sur la surface $R_{n}$, jointes au théorème I, permettent d'énoncer le

THÉORÈME II. - Si l'on choisit sur une surface reglée gauche une trajectoire orthogonale de ses génératrices comine courbe directrice, on peut exprimer la courbure et la torsion géodésiques de toute autre trajectoire orthogonale des génératrices de la surface comme fonctions rationnelles de la forme (3.9) de la courbure et de lı torsion géodésiques de la courbe directrice, le coefficient unique $\beta$ qui figure dans ces fonctions étant une constante égale en valeur absolue à la distance constante des dewx points de cette trajectoire et de la courbe dire. ctrice situés sur la même génératrice de la surface.

Une conséquence remarquable de ce théorème est la proposition suivante:

Si la courbure et la torsion géodésiques d'une trajectoire orthogonale des génératrices d'une surface reglée gauche vérifient une relation algébrique à coefflcients constants, la courbure et la torsion géodésiques de toute autre trajectoire orthogonale des génératrices de la surface vérifient également une relation algébrique à coefficients constants.

\section{II.}

4. Supposons d'abord que la surface considérée $R$ soit une surface de BertrRand.

Dans ce cas les points de la ligne de striction $C$ de $R$ correspondent aux points de la ligne de striction $C_{1}$ d'une autre surface reglee gauche $R_{1}$ de manière que les normales aux surfaces en chaque couple de points homo. logues de leurs lignes de striction coïncident, les deux surfaces ayant en outre des strictions égales sur leurs génératrices issues de chaque couple de points homologues de ces courbes 5, p. 45).

La surface $R_{1}$ qui évidemment est aussi une surface de BERTRand, appartient necéssairement à l'ensemble $(N)$ associè à $R$, car deux surfaces reglées gauches dont les normales centrales coïncident, appartiennent - com. me on le voit aussitôt - chacune à l'ensemble $(N)$ associé à l'autre.

Or, si $\beta_{1}, \omega_{1}$ sont les valeurs des paramètres $\beta, \omega$ qui fignrent dans l'equation (2.7) de l'ensemble $(N)^{*}$ associe à $R$, auxquelles correspond la surface $R_{1}$ de cet ensemble, qui constitue avec $R$ un couple de surfaces de Bermrand, $\beta_{1}$ est nécessairement $\neq 0$ et, d'après les formules (5.7), les invariants $x, \sigma, \varphi$ de $R$ sont des fonctions de l'are $u$ de sa ligne de striction $O$ 
vérifiant nécessairement la relation

$$
\frac{\cos \left(\varphi-\omega_{1}\right)-\beta_{1}\left(x \cos \omega_{1}-x \sin \omega_{1}\right)}{\sin \left(\varphi-\omega_{1}\right)+\beta_{1}\left(x \sin \omega_{1}+\sigma \sin \omega_{1}\right)}=\frac{\cos \varphi}{\sin \varphi}
$$

pour toutes les valeurs de $u$ correspondant aux points de $C$.

La condition (4.1), qui - com ne il résulte des formules (3.7) est en outre suffisante afin que la surface $R$ soit une surface de Bertrand, à l'aide des (1.5), affecte la forme

$$
x_{n} \sin \omega_{1}+\sigma_{g} \cos \omega_{1}=\frac{\sin \omega_{1}}{\beta_{1}} .
$$

La relation (4.2), dans le cas où la ligne de striction $C$ de $R$ est une ligne de courbure, est vérifiée, si l'on $y$ pose $\omega_{1}=0$ ou $\pi, \beta_{1}$ étant une constante arbitraire $\neq 0$, car, dans ce cas, on a $\sigma_{g}=0$.

Alors la surface $R_{n}$, engendrée par les norimales centrales de $R$, est une surface développable et toutes les surfaces de l'ensemble $(N)$ associé à $R$. dont les génératrices homologues de chaque génératrice de $R$ sont parallèles à cette génératrice, admettent sur ces génératrices la même que $R$ striction. Donc, une surface reglée gauche, dont la ligne de striction est une ligne de de courbure, peut être considérée comme une surface de BERTRAND.

Si les invariants $x, \sigma, \varphi$ de la surface $R$ dont la ligne de striction n'est pas une ligne de courbure, vérifient une relation de la forme (4.1), où $\beta_{1}$, $\sin \omega_{1}, \cos \omega_{1}$ sont des constantes dont les deux premiéres sont $\neq 0$, l'en. semble $(N)$ associè à $R$ ne contient qu' une seule surface constituant avec $R$ un couple de surfaces de Bertrand: La surface $R_{1}$ de cet ensemble, qui correspond aux valeurs $\beta_{1}$, $\omega_{1}$ des paramètres $\beta$, $\omega$ que l'équation (2.7) renferme.

La surface $R_{1}$ est également une surface de Bertrand; p.r conséquent la courbure normale $x_{1 n}$ ef la torsion géodésique $\sigma_{\mathrm{lg}}$ de sa ligne de striction $G_{1}$ doivent vérifier une relation de la forme (4.2);

$$
\gamma_{1 n} \sin \omega^{\prime}+\sigma_{1 g} \cos \omega^{\prime}=\frac{\sin \omega^{\prime}}{\beta^{\prime}},
$$

où $\beta^{\prime}, \omega^{\prime}$ sont les valeurs des paramétres $\beta, \omega$ qui figurent dans I'équation la forme (2.7) de l'ensemble $(N)$ associé à la surface $R_{1}$, auxquelles corre. spond la surface $R$ considérée comme une surface appartenant à cet ensemble. Mais on a, comme on le voit aussitôt, $\beta^{\prime}=-\beta_{1}, \omega^{\prime}=-\omega_{1}$. Donc $x_{1 n}, \sigma_{1 g}$ doivent vérifier la relation

$$
x_{1 n} \sin \omega_{1}-\sigma_{1 g} \cos \omega_{1}=-\frac{\sin \omega_{1}}{\beta_{1}},
$$


D'après (4.2), pour que la surface considérée $R$ soit une surface de Bentrand, il faut que la courbure normale $x_{n}$ et la torsion géodésique $\sigma_{g}$ de sa ligne de striction $C$ vérifient une relation de la forme

$$
A x_{n}+B \sigma_{g}=C,
$$

les coefficients $A, B, C$ qui $y$ figurent étant des constantes dont la première et la troisième sont toutes les deux soit $\neq 0$ soit $=0$, la seconde étant, dans ce cas, $\neq 0$.

Cette condition est en outre suffisante afin que $R$ soit une surface de BermRAND.

En effet, si $A=C=0, B \neq 0$, la ligne de striction de $R$ est une ligne de courbure; par conséquent $R$ peut être considérée comme une surface de Bertrand.

Si $A \neq 0, C \neq 0$, en posant

$$
\frac{A}{\sqrt{A^{2}+B^{2}}}=\sin \omega_{1}, \frac{B}{\sqrt{A^{2}+B^{2}}}=\cos \omega_{1}, \frac{A}{C}=\beta_{1},
$$

on peut donner à la relation (4.4) la forme (4.2) qui, étant équivalente à la relation (4.1), est suffisante pour que $R$ soit une surface de BerTrand.

On peat done énoncer le

THÉonìme III. - Afin qu'une surface reglée gauche soit une surface de Bertrand, il faut et il suffit que la courbure normale et la torsion géodésique de sa ligne de striction vérifient une relation linéaire à coefficients constants dont le premier et le troisième sont tous les deux ou bion $\neq 0$ ou bien $=0$, le second étant, dans ce cas, $\neq 0$.

Il est à noter que dans le cas où la ligne de striction $C$ d'une surface de Bertrand $R$ n'est pas une ligne de courbure, de la relation (4.2) à laquelle doivent satisfaire la courbure normale $x_{n}$ et la torsion géodésique $\sigma_{g}$ de la courbe $C$, qui peut s'écrire

$$
\sin \omega_{1}\left(1-\beta_{1} x_{n}\right)=\beta_{1} \cos \omega_{1} \sigma_{g},
$$

où $\beta_{1} \neq 0, \sin \omega_{1} \neq 0$, on déduit que l'on a

$$
\sin \omega_{1}=\frac{\beta_{1}^{2} \sigma_{g}^{2}}{\left(1-\beta_{1} \alpha_{n}\right)^{2}+\beta_{1}^{2} \sigma_{g}^{2}} .
$$

Par ailleurs, d'après la seconde formule (3.8), la torsion géodésique $\sigma_{1 g}$ de la ligne de striction $C_{1}$ de la surface $R_{1}$ qui constitue avec $R$ un couple de Bertrand, est liée avec $x_{n}, \sigma_{g}$ par la relation

$$
\sigma_{1 g}=\frac{\sigma_{g}}{\left(1-\beta_{1} x_{n}\right)^{2}+\beta_{1}^{2} \sigma_{g}^{2}} .
$$


Des deux relations (4.5) et (4.6) on déduit aussitôt que les torsions géodé. siques $\sigma_{g}, \sigma_{1 g}$ des lignes de striction des surfaces $R, R_{1}$ en leurs points situés sur la même génératrice de la surface engendrée par les normales centrales communes des deux surfaces vérifient la relation

$$
\sigma_{g} \sigma_{1 g}=\frac{\sin ^{2} \omega_{1}}{\beta_{1}^{2}}
$$

Remarque. - Les lignes de striction des deux surfaces reglées gauches $R, R_{1}$ qui constituent un conple de Bertrand, d'après un théorème connu (6, p. 143), sont des géodésiques de ces surfaces, dans le cas particulier où ces courbes sont en plus des trajectoires isogonales des génératrices des deux surfaces ou - ce qui revient au même - dans le cas où les deux surfaces sont des surfaces à striction constante. Alors la normale centrale commune des deux surfaces en chaque couple de points homologues de leurs lignes de striction est nécessairement la normale principale commune de ces courbes aux mêmes points. Donc, dans ce cas, les lignes de striction des deux surfaces constituent un couple de courbes de BERTRAND et la relation (4.7) se réduit à la relalion qui - comme on sait $(6, \mathrm{p} .35)$ - est verifiée par les torsions des courbes d'un tel couple en leurs points situés sur la même génératrice de la surface engendrée par les normales principales communes de ces courbes.

5. Si les lignes de striction $C, C_{1}$ d'un couple de surfaces $R, R_{1}$ de Bertrand ne sont pas de lignes de conrbure de ces surfaces, leurs courbures normales $x_{n}, x_{1 n}$ et leurs torsions géodésiques $\sigma_{g}, \sigma_{1 g}$ doivent satisfaire - comme nous l'avons reconnu dans le paragraphe précédent - à deux relations de la forme (4.2) et (4.3), les constantes $\beta_{1}$, $\omega_{1}$ pui $y$ figurent, étant les valeurs des paramètres $\beta$, $\omega$ que l'équation (2.7) de l'ensemble $(N)$ associé à $R$ renferme, auxquelles correspond la surface $R_{1}$.

Par ailleurs, si $R^{*}$ est une surface de cette ensemble correspondant anx valeur's $\beta^{*}, \omega^{*}$ des paramètres $\beta$, $\omega$, la courbure normale $x_{n}^{*}$ et la torsion géodésique $\sigma_{g}^{*}$ de la ligne de striotion $C^{*}$ de $R^{*}$, d'après (3.8), sont liées avec la courbure normale $x_{n}$ et la torsion géodésique $\sigma_{g}$ de la ligne de striction $C$ de $R$ par les relations

$$
x_{n}^{*}=\frac{x_{n}-\beta^{*}\left(x_{n}^{2}+\sigma_{g}^{2}\right)}{\left(1-\beta^{*} x_{n}\right)^{2}+\beta^{* 2} \sigma_{g}^{2}}, \sigma_{g}^{*}=\frac{\sigma_{g}}{\left(1-\beta^{*} x_{n}\right)^{2}+\beta^{* 2} \sigma_{g}^{2}} .
$$

L'élimination des $x_{n}, \sigma_{g}$ entre les relations (5.1) et (4.2) conduit à la relation

$$
\sin \omega_{1}\left\{\left(\beta^{* 2}-\beta^{*} \beta_{1}\right)\left(x_{n}^{* 2}+\sigma_{g}^{*_{2}}\right)+\left(2 \beta^{*}-\beta_{1}\right) x_{n}^{*}+1\right\}-\beta_{1} \cos \omega_{1} \sigma_{g}^{*}=0,
$$


à laquelle doivent satisfaire, dans le cas envisagé, la courbure normale et la torsion géodésique de la ligne de striction de $R^{*}$. Cette relation se réduit à la relation (4.2) ou (4.3), si l'on $y$ pose $\beta^{*}=0$ on $\beta^{*}=\beta_{1}$ respectivement.

D'autre part, si la courbure normale $x_{n}^{*}$ et la torsion géodésique $\sigma_{g}^{*}$ de la ligne de striction $C^{*}$ d'une surface reglée gauche $R^{*}$ vérifient une relation de la forme (5.2) et que l'on ait $\beta_{1} \neq 0$, sin $\omega_{1} \neq 0$, de cette relation, qui peut s'écrire

$$
\sin \omega_{1} \frac{x_{n}^{*}+\beta^{*}\left(x_{n}^{* 2}+\sigma_{g}^{* 2}\right)}{\left(1-\beta^{*} \chi_{n}^{*}\right)^{2}+\beta^{* 2} \sigma_{g}^{* 2}}+\cos \omega_{1} \frac{\sigma_{g}^{*}}{\left(1-\beta^{*} x_{n}^{*}\right)^{2}+\beta^{* 2} \sigma_{g}^{* 2}}=\frac{\sin \omega_{1}}{\beta_{1}},
$$

résulte, eu égard anx (3.6), que l'ensemble $(N)$ associé à la surface $R^{*}$ contient une surface de BerTrand, puisque la courbure normale et la torsion géodésique de sa ligne de striction vérifient une relation de la forme (4.2), ou - ce qui revient au mème - que les normales centrales de $R^{*}$ sont les normales centrales communes d' un couple de surfaces de BeRTrand.

Il s'ensuit que la courbure normale $x_{n}$ ef la torsion géodésique $\sigma_{g}$ de la ligne de striction d'une surface gauche $R$ doivent vérifier une relation de la forme

$$
A\left(\varkappa_{n}^{2}+\sigma_{g}^{2}\right)+B \varkappa_{n}+C \sigma_{g}+D=0
$$

les coefficients $A, B, C, D$ qui $y$ figurent étant des constantes dont an moins la seconde et la quatrième sont $\neq 0$, lorsque la ligne de striction de $R$ n'est pas une ligne de courbure et que ses normales centrales soient les normales centrales communes d'un couple de surfaces de Bertrand.

D'autre part, si la courbure normale et la torsion géodésique de la ligne de striction d'une surface gauche réelle vérifient une relation de la forme (5.3), le second et le quatrième des coefficients constants $A, B, C, D$ qui $y$ figurent, étant $\neq 0$, et que l'on considère les relations

$$
\beta^{* 2}-\beta^{*} \beta_{1}=\frac{A}{D}, 2 \beta^{*}-\beta_{1}=\frac{B}{D}, \beta_{1} \frac{\cos \omega_{1}}{\sin \omega_{1}}=-\frac{C}{\bar{D}},
$$

en éliminant $\beta_{1}$ entre les deux premières, on parvient à la relation

$$
D \beta^{* 2}-B \beta^{*}+A=0
$$

Or, si $4 A D-B^{2} \leq 0$, à chaque racine (réelle) du polynôme $D \beta^{* 2}-B \beta^{*}+A$ on peut faire associer, à l'aide des denx dernières relations (5.4), deux valeurs réelles des $\beta_{1}$, ctg $\omega_{1}$ telles que la relation (5.2), si l'on $y$ remplace $\beta_{1}$, ctg $\omega_{1}$ par ces valeurs et $\beta^{*}$ par la racine considérée, se ramène à la relation 
(5.3); ce qui prouve - comme nons l'avons déjà reconnu - que les normales centrales de $R^{*}$ sont les normales centrales communes d'un couple de surfaces de Bertrand.

On peut done formuler le

THÉnьмe IV. - Afin que les normales centrales d'une surface reglée gauche réelle, dont la ligne de striction n'est pas une ligne de courbure, soient les normales centrales communes d'un couple de surfaces réelles de BERTRAND, il faut et il suffit que la courbure normale et la torsion géodésique de sa ligne de striction vérifient une relation algébrique du second degré de la forme (5.3), les coefficients $A, B, C, D$ qui y figurent élant des constantes telles que l'on ait $4 A D-B^{2} \leq 0$, tandis que $B$ et $D$ sont $\neq 0$.

Il est à noter que, comme il résulte des considérations précédentes, les normales centrales communes d'un couple de surfaces de BERTRAND sont en même temps les normales centrales communes d'un second couple de surfaces de cette espèce, qui en général ne coüncide pas avec le premier.

Par ailleurs, si l'on tient compte du fait que la ligne de striction $C^{*}$ de Ia surface considérée $R^{*}$ de l'ensemble $(N)$ associé à $R$ est une trajectoire orthogonale choisie au hasard des génératrices de la surface $R_{n}$ engendrée par les normales centrales de $R$, on déduit de la relation (5.2) et de la remarque finale dn paragraphe 1 , que, lorsque $R$ est une surface de Bertrand, dont la ligne de striction $C$ n'est pas une ligne de courbure et que l'on choisisse $C$ comme courbe directrice sur la surface $R_{n}$, la courbure et la torsion géodésiques $x_{g}^{\prime}, \sigma_{g}^{\prime}$ de chaque trajectoire orthogonale des génératrices de $R_{n}$ vérifient une relation de la forme

$$
A\left(\beta^{2}-\beta_{1}(\beta)\left(x_{g}^{2}+\sigma_{g}^{\prime 2}\right)+\left(2 \beta-\beta_{1}\right) x_{g}^{\prime}+1\right\}+B \beta_{1} \sigma_{g}^{\prime}=0,
$$

les coefficients $A, B, \beta_{2}, \beta$ qui $y$ figurent étant des constantes dont les trois premières sont les mèmes pour toutes ces courbes, la première et la troisième sont $\neq 0$ et la quartième varie de l'une à l'autre de ces courbes.

On a done le

THÉoneme V. - La courbure et la torsion géodésiques de chaque traje. ctoire orthogonale des génératrices de la surface $R_{n}$ engendrée par les normales centrales communes d'un contple de surfaces de Bertrand, dont les lignes de striction ne sont pas de lignes de courbure, vérifient une relation algébrique du second degré de la forme

$$
A^{\prime}\left(x_{g}^{\prime 2}+\sigma_{g}^{\prime 2}\right)+B^{\prime} x_{g}^{\prime}+C^{\prime} \sigma_{g}^{\prime}+D^{\prime}=0
$$

$\grave{a}$ coefficients qui sont constants sur elle, mais qui varient de l'une à l'autre de ces courbes. Cette relation devient linéaire sur la ligne de striction de cha- 
cune des surfaces dont les normales centrales communes sont les génératrices de la surface.

De ce théorème joint au fait que les normales principales communes d'un conple de courbes de Bermrand sont - comme on le reconnait aisément les normales centrales communes de $\infty^{1}$ couples de surfaces de Bertrand à striction constante, on déduit que la courbure et la torsion géodésiques de chaque trajectoire orthogonale des génératrices de la surface engendrée par les normales principales communes d' un couple de courbes de BERTRAND vérifient une relation algébrique du second degré de la forme (5.6), à coefficients qui sont constants sur elle mais qui varient de l'une à l'autre de ces courbes. Cetle relation sur chaque courbe du couple se reduit à la relation linéaire vérifiée par la courbure et la torsion de cette courbe.

6. Supposons maintenant que la ligne de striction $C$ de la surface considérée $R$ soit une ligne asymptotique de cette surface et, par conséquent, que l'on ait en chaque point de $C$

$$
x_{n}=0
$$

Dans ce cas la courbe $C$ est une góodésique de la surface $R_{n}$ engendrée par les normales centrales de $R$, puisque les surfaces, $R, R_{n}$ se coupent sous un angle droit le long de cette courbe; aussi les normales centrales de $R$ sont-elles les binormales de la conrbe $C$.

En ontre, dans ce cas, les formules (3.8), qui expriment la conrbure normale $x_{1 n}$ et la torsion géodésique $\sigma_{1 g}$ de la ligne de striction $C_{1}$ d'une surface $R_{1}$ de l'ensemble $(N)$ associé à $R$ comme fonctions de la courbure normale $x_{n}$ et de la torsion géodésique $\sigma_{g}$ de la ligne de strietion $C$ de $R$, en verta de $(6.1)$, deviennent

$$
x_{1 n}=-\frac{\beta_{1} \sigma_{g}^{2}}{1+\beta_{1}^{2} \sigma_{g}^{2}}, \sigma_{1 g}=\frac{\sigma_{g}}{1+\beta_{1}^{2} \sigma_{g}^{2}} .
$$

En éliminant $\sigma_{g}$ entre ces deux relations on déduit que la courbure normale $x_{1 n}$ et la torsion geodésique $\sigma_{1 g}$ de la ligne de striotion $C_{1}$ d'une surface $R_{1}$ de l'ensemble $(N)$ associé à la surface $R$, lorsque les normales centrales de $R$ sont les binormales de sa ligne de strietion $C$, doivent vérifier une relation de la forme

$$
\beta_{1}\left(x_{1 n}^{2}+\sigma_{1 g}^{2}\right)+x_{1 n}=0
$$

le coefficient $\beta_{1}$ qui $y$ figure étant égal en valeur absolue à la distance constante, d'après (2.8), des deux points des courbes $C, C_{1}$ situés sur la même normale centrale de $R$. 
D'autre part, les normales centrales d'une surface reglée gauche $R_{1}$ sont les binormales d' une courbe gauche, lorsque la courbure normale et la torsion géodésique de sa ligne de striction. $C_{i}$ vérifient une relation de la forme (6.3).

En effet de cette relation résulte, à l'aide de la première formule (3.8), que l'ensemble $(N)$ associé à la surface $R_{1}$ contient nne surface $R$ dont la ligne de striction est une ligne asymptotique. Par conséquent les normales centrales communes des surfaces $R, l_{1}$ sont les binormales de cette courbe.

On a done le

THÉoRème VI. - Afin que les normales centrales d'une sulface reglée gauche soient les binormales d'une courbe gauche, il faut et il suffit que la courbure normale et la torsion géodésique de sa ligne de striction vérifient une relalion algébrique du second degré de la forme $(6.3)$, le coefficient unique, qui y figure, étant une constante.

La condition (6.3) qui, d'après le théorème VI, est nécesraire et suffisante afin qui une surface reglée gauche jouisse de la propriété indiquée, si l'on $y$ remplace $x_{1 n}$, $\sigma_{l_{g}}$ par leurs valears comme fonction des invariants fondamentaux de la surface: $\chi_{1}, \sigma_{1}, \varphi_{1}$, affecte la forme

$$
\beta_{1}\left(x_{1}^{2}+\sigma_{1}^{2}\right)+x_{1} \cos \gamma_{1}-\sigma_{1} \sin \varphi_{1}=0
$$

et sous cette forme elle est donnée par E. KRUPPA (4, p. 165).

Par ailleurs la courbure normale $x_{1 n}$ et la torsion géodésique $\sigma_{1 g}$ de la ligne de striction $C_{1}$ de la surface $R_{1}$, d'après ce qui est exposé dans la remarque finale $d u$ paragraphe 1 , sont respectivement égales à la courbure et à la torsion géodésiques $x_{1 g}^{\prime}, \sigma_{1 g}^{\prime}$ de cette courbe, considérée comme une courbe tracée sur la surface $R_{n}$ engendrée par les normales centrales communes des surfaces $R, R_{1}$. Donc, d'après (6.3), $x_{1 g}^{\prime}, \sigma_{1 g}^{\prime}$ doivent vérifier la relation

$$
\beta_{1}\left(\chi_{1 g}^{\prime 2}+\sigma_{1 g}^{\prime 2}\right)+\chi_{i g}^{\prime}=0 .
$$

Mais $R_{1}$ est une surface de l'ensemble $(N)$ associé à $R$ choisie au hasard; par conséquent, sa ligne de striction $C_{1}$ est une trajectoire orthogonale des génératrices de la surface $R_{n}$ choisie aussi an hasard. En outre chaque trajectoire orthogonale des génératrices de la surface engendrée par les binormales d' une courbe gauche, d'après le thèorème I, est la ligne de striction de $\infty^{1}$ surfaces reglées gauches qui admettent les binormales de cette courbe comme normales centrales. On peut done énoncer le

Théneme VII. - La courbure et la torsion géodésiques de chaque trajectoire orthogonale des géneratrices de la surface engendrée par les binormales d'une courbe gauche $C$ vérifient une relation algébrique du second degré de la forme (6.4), le coefficient unique qui $y$ fiyure étant une constante égale en 
valeur absolue à la distance constante des deux points de cette trajectoire et de la courbe $C$ situés sur la mêne génératrice de la surface.

De ce théolème, si l'on tient compte de la propriété carastéristique des courbes de Mannheim, d'après laquello les normales principales d' une courbe de cette espèce $C_{1}$ sont les binormales d'une autre conrbe gauche $C$ ef $\mathrm{du}$ fait que la courbe $C_{z}$ est une ligne asymptotique de la surface engendrée par ses normales principales, on déduit que la courbure et la torsion géodésiques de chaque trajectoire orthogonale des génératrices de la surface engendrée par les normales principales d'une courbe de MaNNHEM $C_{1}$ vérifient une réla. tion algébrique du second degrée de la forme (6.4), le coefficient uniqne qui $y$ figure étant égal en valeur absolue à la distance constante des deux points de cette trajectoire et de la courbe dont les binormales sont les normales principales de la courbe $C_{1}$ situés sur la même génératrice de la surface. Cette relation se réduit sur la courbe $C_{1}$ à la relation vérifiée par la courbure et la torsion de cette courbe.

7. Considérons maintenant la surface développable $R_{d}$ tangente à la surface considerée $R$ tout le long de sa ligne de striction $C$.

Les génératrices de la surface $R_{d}$ sont les tangentes à la surface $R$ aux points de la courbe $C$, conjuguées aux tangentes à $R$ en ces mêmes points et - comme on sait (2, p. 189) - la génératrice de cette surface issue du point courant $K(u)$ de $C$ est parallèle au vecteur

$$
\vec{d}=\sigma \bar{e}+x \bar{z},
$$

où $x, \sigma$ sont la courbure et la torsion de $R$ sur sa génératrice issue du point $K$.

Si l'on désigne par $\psi$ l'angle $(\bar{t}, \vec{d})$, où $\vec{t}$ est le vecteur unitaire (1.4) parallèle à la tangente à $C$ en $K$, en vertu des (1.4) et (7.1), on a

$$
\cos \psi=\frac{x \sin \varphi+\sigma \cos \varphi}{\sqrt{x^{2}+\sigma^{2}}}
$$

De cette formule, qui, à l'aide des (1.5), acquiert la forme

$$
\cos \psi=\frac{\sigma_{g}}{\sqrt{x_{x_{m}^{2}}^{2}+\sigma_{g}^{2}}}
$$

on déduit aussitôt que, pour que la ligne de striction d'une surface reglée gauche $R$ soit une trajectoire isogonale des génératrices de la surface développable $R_{d}$ tangente à $R$ tout le long de la courbe $C$, il faut et il suffit que la courbure normale et la torsion géodésique de cette courbe vérifient 
une relation de la forme

$$
A x_{n}+B \sigma_{g}=0
$$

les coefficients $A, B$ qui $y$ figurent étant des constantes dont an moins une est $\neq 0$. On peut donc distinguer trois ens suivant que l'on a $A=0, B \neq 0$, ou $A \neq 0, B=0$, ou $A \neq 0, B \neq 0$.

Si $A=0, B \neq 0$, la ligne de striction $C$ de $R$ est une ligne de courbure, puisque en chaque point de $C$ on a $\sigma_{g}=0$. Dans ce cas la surface $R_{n}$ engen. drée par les normales centrales de $R$ est développable et les trajectoires orthogonales des génératrices de $R_{n}$ sont des lignes de courbure de cette surface. Par conséquent leur torsion géodessique est partout $=0$.

Si $A \neq 0, B=0$, la ligne de striction $C$ de $R$ est une ligne asymptotique de cette surface et ce cas est discouté dans le paragraphe précédent.

Si enfin $A \neq 0, B \neq 0$, en eliminant $x_{n}, \sigma_{g}$ entre la relation (7.3) et les deux relations (3.8) vérifiées par les courbures normales $\chi_{n}, \chi_{1 n}$ et les torsions géodésiques $\sigma_{g}, \sigma_{1 g}$ de la ligne de striction $C$ de $R$ et de la ligne de striction $C_{1}$, d'une surface $R_{I}$ de l'ensemble $(N)$ associé à $R$, on déduit que la cour. bure normale $x_{1 n}$ et la torsion géodésique $\sigma_{1 g}$ de la courbe $C_{1}$ doivent vérifier la relation

$$
\beta_{1} A\left(\varkappa_{1 n}^{2}+\sigma_{1 g}^{2}\right)+A x_{1 n}+B \sigma_{1 g}=0
$$

où $\beta_{1}$, est une constante égale en valeur absolue à la distance constante, d'après (2.8), des deux points des courbes $C, C_{1}$ situés sur la même normale centrale commune des surfaces $R, R_{1}$.

Les normales centrales de la surface $R$ sont en mème temps les normales à la surface développable $R_{d}$ tangente à $R$ tout le long de sa ligne de strietion $C$. Par ailleurs, d'après le théorème $I$, une trajectoire isogonale des génératrices d' nne surface développable est la ligne de striction de $\infty^{1}$ sur. faces reglées gauches qui admettent les normales à la surface aux points de cette courbe comme normales centrales.

Cela étant, de la relation (7.4) on déduit que la courbure normale $x_{n}$ et la torsion géodésique $\sigma_{g}$ de la ligne de striction d' une surface reglée gauche doivent vérifier une relation de la forme

$$
A_{1}\left(\chi_{n}^{2}+\sigma_{g}^{2}\right)+B_{1} \chi_{n}+C_{1} \sigma_{g}=0
$$

les coefficients $A_{1}, B_{1}, C_{1}$ qui $y$ figurent étant des constantes, dont au moins les deux dernières sont $\neq 0$, lorsque les normales centrales de la surface sont les normales à une surface développable aux points d' une trajectoire isogonale - non orthogonale - de ses génératrices. 
D'autre part, la condition (7.5) vérifiée par la courbure normale et la torsion géodésique de la ligne de striction d'une surface reglée gauche $R$ est suffisante, afin que les normales centrales de la surface soient les normales à une surface développable aux points d'une trajectoire isogonale de ses génératrices, car de cette relation, qui, si l'on $y$ pose $B_{1}=A, A_{1}=B_{1} \beta_{1}$, $C_{1}=B B_{1}$, acquirert la forme

$$
A\left\{\beta_{1}\left(x_{n}^{2}+\sigma_{g}^{2}\right)+x_{n}\right\}+B \sigma_{z}=0
$$

on déduit, à l'aide des formules (3.8), que la courbure normale et la torsion géodésique de la ligne de striction d' une surface de l'ensemble $(N)$ associé à $R$ vérifient une relation de la forme (7.3). Cette surface coïncide avec $R$, si $A_{1}=0$.

On peut done énoncer le

Théonìm VIIT - Afin que les normales centrales d' une surface reglée gauche soient les normales à une surface déreloppable aux points d'une traje. ctoire isogonale - non orthogonale - de ses génératrices, il faut et il suffit que la courbure normale et la torsion géodésique de sa ligne de striction véri. fient une relalion algébrique du second degré de la forme (7.5), les coefficients qui y figurent étant des constantes dont au moins les deux dernières sont $\neq 0$.

En outre, d'après la remarque finale du paragraphe 1, la conrbure et la torsion géodésiques $x_{1 g}^{\prime}, \sigma_{1 g}^{\prime}$ de la ligne de striction $C_{1}$ d'une surface $R_{1}$ de l'ensemble $(N)$ associé à $R$, considérée comme une courbe tracée sur la sur face $R_{n}$ engendrée par les normales centrales de $R$, doivent vérifier, dans le cas envisagé, la relation

$$
A \beta_{1}\left(\varkappa_{1 g}^{\prime 2}+\sigma_{1 g}^{\prime 2}\right)+A \chi_{1 g}^{\prime}+B \sigma_{1 g}^{\prime}=0
$$

à laquelle on parvient en remplaçant dans la relation (7.4), $x_{1 n}, \sigma_{1 g}$ par $x_{1 g}^{\prime}$, $\sigma_{1 g}^{\prime}$ respectivement. Les coefficients $A, B$ qui figurent dans la relation (7.6) sont les coefficients de la relation (7.3), tandis que $\beta_{1}$ est une constante egale en valeur absolue à la distance constante, d'après (2.8), des deux points de la courbe $C_{1}$ et de la ligne de striotion $C$ de $R$, situés sur la même génératrice de la surface $R_{n}$.

Cette constatation, jointe au fait que, d'après le théorème $I$, chaque trajectoire orthogonale des génératrices de la surface $R_{n}$ engendrée par les normales à une surface développable aux points d'une trajectoire isogonale de ses génératrices, est la ligne de striction de $\infty^{1}$ surfaces reglées gauches qui admettent les génératrices de $R_{n}$ comme normales centrales, permet de formuler le

THÉORk̀m IX. - La courbuve et la torsion géodésiques de chaque traje. ctoire orthogonale des génératrices de la surface engendrée par les normales à 
une surface développable aux points d'une trajectoire isogonale $C$ - non orthogonale - de ses génératrices, vérifient une relation algébrique du second degrê de la forme (7.6) à coefficients qui sont constants sur elle. Les deux pre. miers des coefficients $A, B, \beta_{1} q u i$ figurent dans celte relation, étant tous les deux $\neq 0$, sont les mêmes sur toutes ces courbes, tandis que le troisième varie de l'une à l'autre. Cette relation se réduit sur la courbe $C$ à la relation linéaire et homogène vérifiée par la courbure et la torsion géodésiques de cette courbe.

De ce théoreme, si l'on tient compte du fait que les normales prinoipales d'une hélice cylindrique sont les normales le long de cette courbe à la sur. face cylindrique dont l'bélice est une géodésique, on déduit que la courbure et la torsion géodésiques de chaque trajectoire orthogonale des génératrices de la surface engendrée par les normales principales d'une hélice cylindrique, sont liées par une relation algébrique du second degré de la forme (7.6), les coefficients $A, B$, $\beta$ qui y figurent étant constants sur elle. Le coefficient $\beta$ varie de l'une à l'autre de ces courbes, tandis que les dewx premiers sont invariables; ce sont les coefficients qui figurent dans la relation linéaire et homogène vérifiée par la courbure et la torsion de l'hélice.

8. Soit $R_{3}$ une surface de l'ensemble $(N)$ associé à la surface considérée $R$, correspondant aux valeurs $\beta_{1}, \omega_{1}$ des paramètres $\beta, \omega$ qui figurent dans l'équation (2.7) de cet ensemble.

La courbure normale $x_{1 n}$ et la torsion géodésique $\sigma_{1 g}$ de la ligne de strietion $C_{1}$ de $R_{1}$ sont les fonctions (3.8) de la courbure normale $\kappa_{\pi}$ et de la torsion géodésique $\sigma_{g}$ de la ligne de $C$ striction $C$ de $R$.

En éliminant $\beta_{1}$ entre les deux relations (3.8), on parvient facilement à la relation

$$
\frac{\sigma_{1 g}}{\chi_{1 n}^{2}+\sigma_{1 g}^{2}}=\frac{\sigma_{g}}{\chi_{n}^{2}+\sigma_{g}^{2}} ;
$$
ce qui montre que le rapport $\frac{x_{n}^{2}+\sigma_{g}^{2}}{\sigma_{g}}$ est le même pour toutes les surfaces
de l'ensemble $(N)$ associè à $R$.

La valeur de ce rapport an point courant $K(u)$ de la ligne de striction $C$ de $R$ est égale au paramètre de distribution $p_{n}$ de la surface $R_{n}$, engen. drée par les normales centrales de $R$ sur sa génératrice issue de ce point.

Eu effet, d'apròs la formule connue, le paramòtre de distribution $p_{n}$ de $R_{n}$ sur sa génératrice issue du point $K(u)$ de la courbe $C$, est

$$
p_{n}=\frac{\dot{\bar{\rho}} \wedge \bar{n}) \times \dot{\bar{n}}}{\dot{\bar{n}}^{2}}
$$


Cette formule, si l'on $y$ remplace $\dot{\bar{\rho}}, \bar{n}$ par leurs valeurs (1.4) et (1.2), acquiert la forme

$$
p_{n}=\frac{x \sin \varphi+\sigma \cos \varphi}{x^{2}+\sigma^{2}}
$$

ou finalement, à l'aide des (1.5),

$$
p_{n}=\frac{\sigma_{g}}{x_{n}^{2}+\sigma_{g}^{2}} .
$$

De cette formule on déduit aussitôt que, pour que la surface $R_{n}$ engendrée par les normales centrales d'une surface reglée, soit une surface à paramètre de distribution constant, il faut et il suffit que l'on ait

$$
a\left(x_{n}^{2}+\sigma_{g}^{2}\right)-\sigma_{g}=0
$$

où $a$ est une constante.

On a done le

ThÉonìme $X$. - Pour que la surface engendrée par les normales centrales d'une surface reglée gauche $R$, soit une surface à paramètre de distribution constant, il faut et il suffit que la courbure normale et la torsion géodésique de la ligne de striction de $R$ vérifient une relation algébrique du second degré de la forme (8.3), le coefficient unique qui y figure étant égal au paramètre de distribution constant de cette surface.

Par ailleurs des deux relations (8.1) et (8.3) on déduit aussitôt, si l'on tient compte en outre de la remarque finale du paragraphe 1, que la courbure et le torsion géodésiques $\chi_{1 g}^{\prime}, \sigma_{1 g}^{\prime}$ de la ligne de striction $C_{1}$ d'une surface $R_{1}$ de l'ensemble $(N)$ associé à $R$, considérée comme une courbe tracée sur la surface $R_{n}$ engendrée par les normales centrales de $R$ verifient la relation

$$
a\left(x_{1 g}^{\prime 2}+\sigma_{1 g}^{\prime 2}\right)-\sigma_{1 g}^{\prime}=0
$$

où $a$ est une constante, lorsque $R_{n}$ est une surface à paramètre de distribu. tion constant égal à $a$.

Cette constatation jointe au fait que, d'après le théorème $I$, les génératrices d'une surface reglée à paramètre de distribution constant sont les normales centrales communes de $\infty^{2}$ surfaces reglées gauches, permet de formuler le

THÉonème XI. - La courbure el la torsion géodésiques de chaque trajecloire orthogonale des génératrices d' une surface reglée à paramètre de distribution constant vérifient une relation algébrique du second degré de la forme (8.4), le coefficient unique qui y figure étant égal au paramètre de distribution constant de la surface. 
9. Supposons enfin que la ligne de striction $C$ de la surface $R$ soit une courbe de cette surface, dont la courbure normale et la torsion géodé. sique sont toutes les deux constantes:

$$
x_{n}=c_{1}, \sigma_{g}=c_{2}
$$

où $c_{I}, c_{2}$ sont des constantes dont au moins la seconde est $\neq 0$.

Dans ce cas, d'après ce qui est exposé dans le paragraphe 7, la courbe $C$ est une trajectoire isogonale des génératrices de la surface développable $R_{d}$ tangente a $R$ le long de cette courbe.

En outre, les normales centrales do $R$, d'après ce qui est exposé dans le paragraphe 6 , sont les binormales d'une courbe gauche $C_{1}$ : la ligne de striction la surface $R_{1}$ de l'ensemble $(N)$ associé à $R$ correspondant aux valeurs $\beta_{1}=\frac{c_{2}}{c_{1}^{2}+c_{2}^{2}}$, $\omega_{1}$ des paramètre $\beta, \omega$ qui figurent dans l'équation (2.7) de cet ensemble.

Par ailleurs, dans ce cas, la surface $R_{n}$ engendrée par les normales centrales de $R$, grâce aux (9.1), est une surface à paramètre de distribution constant:

$$
p_{n}=\frac{c_{2}}{c_{1}^{2}+c_{2}^{2}} \neq 0
$$

Cela étant, d'après le théorème XI, la courbure et la torsion géodésiques $x_{i g}^{\prime}, \sigma_{1 g}^{\prime}$ de la courbe $C_{1}$ dont les binormales sont les normales centrales de $R$, considérée comme une courbe de la surface $R_{n}$, doivent vérifier la relation

$$
\left.p_{n}^{(} x_{1 g}^{\prime 2}+\sigma_{1 g}^{\prime 2}\right) \cdots \sigma_{1 g}^{\prime}=0 .
$$

Mais la courbe $C_{1}$ est nécessairement nne géodésique de la surface $R_{n}$ engendrée par ses binormales. On aura done $x_{1 g}^{\prime}=U, \sigma_{1 g}^{\prime}=\sigma_{1}$, où $\sigma_{1}$ est la torsion de cette courbe et de la relation $(9.3)$ on déduit que l'on doit avoir soit $\sigma_{1 g}^{\prime}=\sigma_{1}=0$ soit $\sigma_{1 g}^{\prime}=\sigma_{1}=\frac{1}{p_{n}} \neq 0$.

Si $\sigma_{1}=0$, la courbe $C_{1}$ serait une courbe plane et la surface $R_{n}$ engen. drée par ses binormales serait une surface cylindrique, ce qui, d'après l'hypothèse faite pour le paramètre de distribution de $R$, est exclu.

Done, dans le cas envisagé, les normales centrales de la surface $R$ sont les binormales d'une courbe gauche à torsion constante:

$$
\sigma_{1}=\frac{1}{p_{n}}=\frac{c_{1}^{2}+c_{1}^{2}}{c_{2}}
$$


D'autre part, la surface $R_{b}$ engendrée par les binormales d'une courbe gauche $C$ à torsion constante $c$ est - comme on sait (1, p. 104) - une surface gauche à paramètre de distribution constant $p_{n}=\frac{1}{c}$.

Or, si l'on choisit sur la surface $R_{b}$ la courbe $C$ comme courbe directrice et que l'on tienne compte du fait que la courbure et la torsion géodésiques de $C$ considerée comme une courbe tracée sur $R_{b}$, sont

$$
x_{1 g}^{\prime}=0, \quad \sigma_{1 g}^{\prime}=\sigma_{1}=c,
$$

puisque $C$ est une géolésique de $R_{b}$, on obtient à l'aide des (3.9) et (9.5) pour la courbure et la torsion géodésiques $x_{g}^{\prime}, \sigma_{g}^{\prime}$ d' une trajectoire orthogonale des génératrices de la surface $R_{b}$ les expressions

$$
x_{g}^{\prime}=-\frac{\beta^{\prime} \sigma^{2}}{1+\beta^{\prime 2} c^{2}}, \sigma_{g}^{\prime}=\frac{c}{1+\beta^{\prime 2} c^{2}}
$$

ce qui montre que $x_{g}^{\prime}, \sigma_{g}^{\prime}$ sont constantes sur chaque trajectoire orthogonale des génératrices de la surface $R_{b}$, le coefficient $\beta^{\prime}$ qui figure dans les formules (9.6) Etant constant sur chacune de ces courbes.

Les considérations précédentes permettent d'énoncer le

Théontime XII. - La courbure et la torsion géodésiques des trajectoires orthogonales des génératrices d'une surface reglée gauche ne sont constantes sur chacune de ces courbes que dans le cas oì les génératrices de la surface sont les binormales d'une courbe gauche à torsion constante.

Il est à noter que, d'après un théorème du à X. Antomalix (4, p. 168), si une trajectoire orthogonale des génératrices de la surface engendrée par les binormales d'une courbe gauche $C_{1}$ est une courbe de la surface $\dot{a}$ courbure géodésique constante toutes les trajectoires orthogonales des génératrices de la surface sont nécessairement des courbes à courbure géodésique constante.

Dans ce cas la courbe $C_{1}$ est nécessairement une courbe à torsion con. stante.

En effet, $C_{1}$ est nue trajectoire orthogonale des génératrices de la surface $R_{b}$ engendrée par ses binormales et en mêne temps une géodésique de cette surface. On aura donc, en désignant par $\chi_{l_{g}}^{\prime}, \sigma_{I_{g}}^{\prime}$ la courbnre et la torsion géodésiques de $C_{1}, x_{1 g}^{\prime}=0, \sigma_{1 g}^{\prime}=\sigma_{1}$, où $\sigma_{1}$ est la torsion de cette courbe.

$\mathrm{Or}$, si une autre trajectoire orthogonale $C^{\prime}$ des génératrices de $R_{b}$ est une courbe à courbure géodésique constante: $x_{g}^{\prime}=c^{\prime} \neq 0$ et que l'on choisisse sur la surface $R_{b}$ la courbe $C_{1}$ comme courbe directrice, on aura, d'après la première formule (3.9), pour la courbure géodésique $x_{g}^{\prime}$ de la courbe $C^{\prime}$ 
l'expression

$$
x_{g}^{\prime}=-\frac{\beta^{\prime} \sigma_{1}^{2}}{1+\beta^{\prime 2} \sigma_{1}^{2}}=c t e,
$$

ce qut prouve, eu égard au fait que $\beta^{\prime}$ est une constante sur la courbe $C^{\prime}$, que $C_{1}$ doit être, dans le cas envisagé, une courbe à torsion constante.

De cette constatation jointe au théorème XII résulte que, si une trajectoire orthogonale des génératrices de la surface engendrée par les binormales d'une courbe gauche est une courbe de la surface d̀ courbure géodésique constante $(\neq 0)$, la courbure et la torsion géodésiques des trajectoires orthogonales des génératrices de la surface sont sur chacune de ces courbes toules les deux constantes.

\section{BIBLIOGRAPHIE}

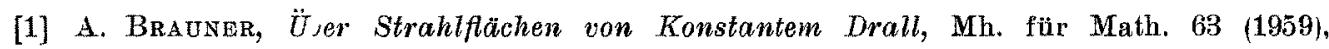
pp. 101-111.

[2] J. KRAMes, Über den Drall windschiefer Fläehen, Anz. der Öst. Akad. der Wiss. Wien, Math-Nat. Klasse 97 (1960), pp. 187-192.

[3] E. Kruppa, Analytische und konstrultive Differential geometrie, Springer 1957.

[4] - - Zur Differentialgeometrie der Strahlfächen und Raumkurven, Stzb. der Öst. Akad. der Wiss. Wien (1957), pp. 143176.

[5] G. Sannia, Una rappresentazione intrinseca delle rigate, Giorn. di Mat. 63 (1925), pp. 31.47 .

[6] G. E. Weatherbura, Differential gesmotry of three dimensions, vol, I, Cambridge, 1861. 